\title{
Strong Dominating Sets of Some Arithmetic Graphs
}

\author{
M.Manjuri \\ Dept. of Applied Mathematics, S.P.Women's \\ University, Tirupati-517502, Andhra Pradesh, India
}

\author{
B.Maheswari \\ Dept. of Applied Mathematics, S.P.Women's \\ University, Tirupati-517502, Andhra Pradesh, India
}

\begin{abstract}
Graph Theory has been realized as one of the most flourishing branches of modern Mathematics finding widest applications in all most all branches of Sciences, Social Sciences, Engineering, Computer Science, etc. Number Theory is one of the oldest branches of Mathematics, which inherited rich contributions from almost all greatest mathematicians, ancient and modern. Using the number theoretic function Euler totient function an Euler totient Cayley graph is defined and in this paper we study the Strong domination parameters of Euler totient Cayley graphs and Arithmetic $\mathrm{V}_{\mathrm{n}}$ Graphs.
\end{abstract}

\section{Keywords}

Euler totient Cayley graphs, Arithmetic $\mathrm{V}_{\mathrm{n}}$ Graphs, Strong domination, Cayley Graph

\section{Subject Classification: 68R10}

\section{INTRODUCTION}

Nathanson [1] was the pioneer in introducing the concepts of Number Theory, particularly, the 'Theory of Congruences' in Graph Theory, thus paved the way for the emergence of a new class of graphs, namely "Arithmetic Graphs". Inspired by the interplay between Number Theory and Graph Theory several researchers in recent times are carrying out extensive studies on various Arithmetic graphs in which adjacency between vertices is defined through various arithmetic functions.

Cayley Graphs are another class of graphs associated with elements of a group. If this group is associated with some Arithmetic function then the Cayley graph becomes an Arithmetic graph. The Cayley graph associated with Euler totient function is called an Euler totient Cayley graph .

\section{EULER TOTIENT CAYLEY GRAPH}

For each positive integer $n$, let $Z_{n}$ be the additive group of integers modulo $n$ and $S$ be the set of all numbers less than $n$ and relatively prime to $n$. The Euler Totient Cayley graph $G\left(Z_{n}, \varphi\right)$ is defined as the graph whose vertex set $\mathrm{V}$ is given by $Z_{n}=\{0,1,2, \ldots n-1\}$ and the edge set is given by $E=\{(x, y) / x-y \in S\}$.

Some properties of Euler totient Cayley graphs can be found in [2] and the domination parameters of these graphs are presented in [3].

The Euler Totient Cayley graph $G\left(Z_{n}, \varphi\right)$ is a complete graph if $n$ is a prime and it is $\Phi(n)-$ regular.

\section{ARITHMETIC $V_{n}$ GRAPH AND ITS PROPERTIES}

Let $n$ be a positive integer such that $n=$ $p_{1}^{\alpha_{1}} p_{2}^{\alpha_{2}} \ldots \ldots \ldots p_{k}^{\alpha_{k}}$. Then the Arithmetic $V_{n}$ graph is defined as the graph whose vertex set consists of the divisors of $n$ and two vertices $u, v$ are adjacent in $V_{n}$ graph if and only if $\operatorname{GCD}(u, v)=p_{i}$, for some prime divisor $p_{i}$ of $n$.
In this graph vertex 1 becomes an isolated vertex. Hence we consider Arithmetic $V_{n}$ graph without vertex 1 as the contribution of this isolated vertex is nothing when domination parameters are studied.

Clearly, $V_{n}$ graph is a connected graph. If $n$ is a prime, then $V_{n}$ graph consists of a single vertex. Hence it is connected. In other cases, by the definition of adjacency in $V_{n}$, there exist edges between prime numbers, their prime powers and also to their prime products. Therefore each vertex of $V_{n}$ is connected to some vertex in $V_{n}$.

It is observed that the domination parameters these graphs are functions of $k$, where $k$ is the core of $n$, that is the number of distinct prime divisors of $n$ [4].

Let $G\left(V_{n}\right)$ denote the $V_{n}$ graph throughout this paper.

\section{Strong domination}

Let $G(V, E)$ be a graph and $u, v \in V$. Then, $u$ strongly dominates $v$ if (i) $u v \in E$ and (ii) $\operatorname{deg} u \geq \operatorname{deg} v$. A set $D \subset V$ is called a strong-dominating set (sd-set) of $G$ if every vertex in $V-D$ is strongly dominated by at least one vertex in $D$. The strong domination number $\gamma_{s}$ of $\mathrm{G}$ is the minimum cardinality of a strong dominating set.

Some results on strong domination can be seen in [5].

In the following sections we find minimum strong dominating sets of $G\left(Z_{n}, \varphi\right)$ and $G\left(V_{n}\right)$ graphs and obtain their strong domination number in various cases.

\section{STRONG DOMINATING SETS OF EULER TOTIENT CAYLEY GRAPH}

Theorem 4.1: If $n$ is a prime, then the strong domination number of $G\left(Z_{n}, \varphi\right)$ is 1 .

Proof: Let $n$ be a prime. Then $G\left(Z_{n}, \varphi\right)$ is a complete graph and hence every vertex is of degree $n-1$. So, any single vertex dominates all other vertices in $G\left(Z_{n}, \varphi\right)$. Hence every vertex in $V-D$ is adjacent with a vertex in $D=\{t\}$, where $t$ is any vertex in $V$. Obviously the degree of every vertex in $V-D$ of $G\left(Z_{n}, \varphi\right)$ is equal to the degree of the vertex $t \in D$ in $G\left(Z_{n}, \varphi\right)$. Thus $D=\{t\}$ is a strong dominating set with minimum cardinality 1 .

$$
\text { Hence } \gamma_{s}\left(G\left(Z_{n}, \varphi\right)\right)=1 \text {. }
$$

Theorem 4.2: The strong domination number of $G\left(Z_{n}, \varphi\right)$ is 2 , if $n=2 p$ where $p$ is an odd prime.

Proof: Let us consider $G\left(Z_{n}, \varphi\right)$ for $n=2 p, \quad p$ is an odd prime. Then the vertex set is given by $=\{0,1,2, . .2 p-1\}$. Let $V$ be decomposed into the following disjoint subsets.

1. The set of odd integers which are less than $n$ and relatively prime to $n$.

But this set is nothing but $S$, since $n=2 p$,

2. The set $M$ of non-zero even integers,

3. The set $D$ of integers 0 and $p$. 
We now show that $D=\{0, p\}$ is a dominating set of $G\left(Z_{n}, \varphi\right)$. By the definition of edges in $G\left(Z_{n}, \varphi\right)$, it is clear that the vertices in $S$ are adjacent with the vertex 0 and hence they are dominated by the vertex 0 .

Now consider the elements of $M$. By the definition of $M$, it contains non-zero even numbers. So if $v \in M$ then $v-p$ is an odd number, because $p$ is an odd prime. Hence $v-p$ or $p-v \in S$. That is the vertex $v$ is adjacent with the vertex $p$. This implies that the vertices of $M$ are dominated by the vertex $p$. Thus every vertex of $V-D$ is dominated by the vertices of $D$. Therefore $D$ is a dominating set of $G\left(Z_{n}, \varphi\right)$. Now we show that $D=\{0, p\}$ is a strong dominating set of $G\left(Z_{n}, \varphi\right)$.

By the properties of $G\left(Z_{n}, \varphi\right)$, we know that the graph $G\left(Z_{n}, \varphi\right)$ is $\varphi(n)-$ regular. So for $n=2 p, \quad G\left(Z_{n}, \varphi\right)$ is $(p-1)$-regular. That is every vertex of $G\left(Z_{n}, \varphi\right)$ is of degree $p-1$.

That is the degree of the vertices $\{0,1,2, \ldots \ldots \ldots . p-1, p+$ $1, \ldots ., 2 p-1\} \in V-D$ of $G\left(Z_{n}, \varphi\right)$ is equal to the degree of vertices in $D=\{0, p\}$ in $G\left(Z_{n}, \varphi\right)$.

So the vertices in $S$ are strongly dominated by the vertex $0 \in D$ and the vertices in $M$ are strongly dominated by the vertex $p \in D$.

Thus every vertex in $V-D$ is strongly dominated by at least one vertex in $D$.

Therefore $D$ is a strong dominating set of $G\left(Z_{n}, \varphi\right)$. Since the graph $G\left(Z_{n}, \varphi\right)$ is $(p-1)$-regular, a single vertex cannot dominate the rest of $p-1$ vertices. Therefore $D=\{0, p\}$ becomes a minimal strong dominating set with cardinality 2 .

$$
\text { Thus } \gamma_{s}\left(G\left(Z_{n}, \varphi\right)\right)=2 \text {. }
$$

Theorem 4.3: Suppose $n$ is neither a prime nor $2 p$. Let $n=p_{1}^{\alpha_{1}} p_{2}^{\alpha_{2}} \ldots \ldots . p_{k}^{\alpha_{k}}$, where $p_{1}, p_{2}, \ldots p_{k}$ are primes and $\alpha_{1}, \alpha_{2}, \ldots . \alpha_{k}$ are integers $\geq 1$. Then the strong domination number of $G\left(Z_{n}, \varphi\right)$ is given by $\gamma\left(G\left(Z_{n}, \varphi\right)\right)=\lambda+1$, where $\lambda$ is the length of the longest stretch of consecutive integers in $V$, each of which shares a prime factor with $n$.

Proof: Suppose $n=p_{1}^{\alpha_{1}} p_{2}^{\alpha_{2}} \ldots \ldots . p_{k}^{\alpha_{k}}$ and $n$ is neither a prime nor 2 p. Let us consider the vertex set $V$ of $G\left(Z_{n}, \varphi\right)$ given by $V=\{0,1,2, n-1\}$. Then the set $V$ falls into the following disjoint subsets.

1. The set $S$ of integers relatively prime to $n$,

2. The set $X=\left\{S_{i}\right\}$, where $S_{i}$ is a collection of consecutive integers in $V$ such that for every $x$ in $S_{i}, \operatorname{GCD}(x, n)>1$

3. The singleton set $\{0\}$.

By the definition of adjacency in $G\left(Z_{n}, \varphi\right)$, it is obvious that the vertices of $S$ are dominated by the vertex 0 . Let $S_{\lambda}$ be a subset in $X$ with maximum cardinality $\lambda$. Suppose $S_{\lambda}=\left\{x_{1}, x_{2}, x_{3}, \ldots ., x_{\lambda}\right\}$ where $\operatorname{GCD}\left(x_{i}, n\right)>1$ for $i=1,2,3, \ldots, \lambda$.

Since $x_{1}, x_{2}, x_{3}, \ldots, x_{\lambda}$ are in consecutive order, we haveGCD $\left(x_{1}-1, n\right)=1, \operatorname{GCD}\left(x_{2}-2, n\right)=1, \ldots \ldots$, $\operatorname{GCD}\left(x_{\lambda}-\lambda, n\right)=1$. Therefore it follows that $x_{1}-1, x_{2}-2, \quad x_{3}-3, \ldots \ldots, x_{\lambda}-\lambda \in S$. So the vertices $x_{1}, x_{2}, \ldots \ldots, x_{\lambda}$ are adjacent with the vertices $1,2, \ldots \ldots, \lambda$ respectively. Hence the vertices in $S_{\lambda}$ are dominated by the vertices $1,2, \ldots \ldots, \lambda$.

In a similar way, we can show that all vertices of each collection $S_{i}$ in $X$ are dominated by the vertices $1,2, \ldots \ldots, \lambda$. Thus the set $D=\{0,1,2, \ldots \ldots, \lambda\}$ becomes a dominating set in $G\left(Z_{n}, \varphi\right)$ as it dominates every vertex in $V-D$. Now we show that $D$ is a strong dominating set of $G\left(Z_{n}, \varphi\right)$.

We know that the graph $G\left(Z_{n}, \varphi\right)$ is $\quad \varphi(n)$ - regular. So, the degree of every vertex in $V-D$ of $G\left(Z_{n}, \varphi\right)$ is equal to the degree of every vertex in $D$. Thus every vertex in $V-D$ is strongly dominated by at least one vertex in $D$.

Therefore $D$ is a strong dominating set of $G\left(Z_{n}, \varphi\right)$

We claim that $D$ is minimal. Suppose we delete 0 from $D$ and let $D^{\prime}=\{1,2, \ldots \ldots, \lambda\}$. Then $D^{\prime}$ cannot be a dominating set of $G\left(Z_{n}, \varphi\right)$, since the vertices of $S$ are dominated only by the vertex 0 and $0 \notin D^{\prime}$

Suppose we delete any other vertex, say $j$ from $\{1,2, \ldots, \lambda\}$. Let $D^{\prime \prime}=\{0,1,2, \ldots, j-1, j+1, \ldots, \lambda\}$. Suppose $D^{\prime \prime}$ is a dominating set of $G\left(Z_{n}, \varphi\right)$. Consider the vertex $x_{j}$ in $S_{\lambda}$, then the vertex $x_{j}$ is dominated by some vertex $k$ in $D^{\prime \prime}$ and $k \neq 0$. This implies that $x_{j}-k \in S$. Now $x_{1}, x_{2}, x_{3}, \ldots ., x_{\lambda}$ are in consecutive order implies that $x_{j}-k=x_{j-k}$. So $x_{j-k} \in S$, for all $j$ and $k$ such that $1 \leq j \leq \lambda$ and $1 \leq k \leq \lambda-1$. For $j=\lambda, k=\lambda-1$, we get $x_{j-k}=x_{1} \in S$, a contradiction because $\operatorname{GCD}\left(x_{1}, n\right)>1$. Therefore it follows that $D^{\prime \prime}$ cannot be a dominating set of $G\left(Z_{n}, \varphi\right)$ and hence $D$ is minimal.

If a minimal dominating set is formed in any other way, then the cardinality of such a set is not smaller than that of $D$. This follows from the properties of the prime divisors of a number.

Hence $D$ becomes a strong dominating set of $G\left(Z_{n}, \varphi\right)$ with minimum cardinality.

Therefore $\gamma_{s}\left(G\left(Z_{n}, \varphi\right)\right)=|D|=\lambda+1$.

\section{STRONG DOMINATING SETS OF ARITHMETIC $\boldsymbol{V}_{\boldsymbol{n}}$ GRAPH}

Theorem 5.1: If $n=p_{1}^{\alpha_{1}} p_{2}^{\alpha_{2}} \ldots \ldots p_{k}^{\alpha_{k}}$, where $p_{1}, p_{2}, \ldots ., p_{k}$ are primes and $\alpha_{1}, \alpha_{2}, \ldots . \alpha_{k}$ are integers $\geq 1$, then the strong domination number of $G\left(V_{n}\right)$ is given by

$$
\gamma_{s}\left(G\left(V_{n}\right)\right)=\left\{\begin{array}{cc}
k-1 & \text { if } \alpha_{\mathrm{i}}=1 \text { for more than one i, } \\
k & \text { otherwise. }
\end{array}\right.
$$

where $k$ is the core of $n$.

Proof: Suppose $n=p_{1}^{\alpha_{1}} p_{2}^{\alpha_{2}} \ldots \ldots . p_{k}^{\alpha_{k}}$. Consider $G\left(V_{n}\right)$ with vertex set $V$. Now we have the following possibilities.

Case 1: Suppose $\alpha_{\mathrm{i}}>1$ for all $i$. Now we show that the set $D=\left\{p_{1}, p_{2}, \ldots ., p_{k}\right\}$ becomes a dominating set of $G\left(V_{n}\right)$.

By the definition of $G\left(V_{n}\right)$ graph, the vertices in $G\left(V_{n}\right)$ are primes $p_{1}, p_{2}, \ldots \ldots, p_{k}$, their powers and their products.

All the vertices $u \in V-D$, for which $\operatorname{GCD}\left(u, p_{i}\right)=p_{i}$ are adjacent with the vertex $p_{i}$ in $D$ for all $i=1,2, \ldots k$. Since every vertex in $V-D$ has atleast one prime factor viz., $p_{1}, p_{2}, \ldots \ldots, p_{k}$ ( as they are divisors of $n$ ), every vertex in $V-D$ is adjacent with at least one vertex in $D$. Thus $D$ becomes a dominating set of $G\left(V_{n}\right)$. 
Now we show that $D$ is a strong dominating set of $G\left(V_{n}\right)$.

By the definition of edges in $G\left(V_{n}\right)$, we see that the degree of $p_{i}^{r},<$ the degree of $p_{i}, i=1,2,3, \ldots, k ; r>1$ and the degree of $p_{i}>$ the degree of $p_{i}^{r} p_{j}^{s}$, the degree of $p_{i}^{r} p_{j}^{s} p_{l}^{t}, \ldots$, the degree of $p_{i}^{r} p_{j}^{S} \ldots \ldots \ldots p_{k}^{u}, r=1,2, \ldots, \alpha_{i}$;

$s=1,2, \ldots, \alpha_{j} ; \ldots ; u=1,2, \ldots \ldots \alpha_{k}$ in $G\left(V_{n}\right)$.

So, the degree of $p_{i} i=1,2, \ldots \ldots, k$ is greater than the degree of all other vertices in $G\left(V_{n}\right)$. Thus every vertex in $V-D$ is strongly dominated by at least one vertex in $D$.

Therefore $D=\left\{p_{1}, p_{2}, \ldots ., p_{k}\right\}$ is a strong dominating set of $G\left(V_{n}\right)$.

We now prove that $D$ is minimal. Suppose we remove any $p_{i}$ from $D$. Then the vertices of the form $p_{i}^{r}$, $r>1$ are not dominated by any other vertex $p_{j}$ in $\mathrm{D}$ as $\operatorname{GCD}\left(p_{i}^{r}, p_{j}\right)=1$ for $i \neq j$. Therefore every $p_{i}$,

$i=1,2, \ldots \ldots, k$ must be included into $D$.

If we form a minimum dominating set in any other manner, then the order of such a set is not smaller than that of $D$. This follows from the properties of prime divisors of a number. Hence $\gamma_{s}\left(G\left(V_{n}\right)\right)=k$.

Case 2: Suppose $\alpha_{\mathrm{i}}=1$ for only one $i$. That is, $p_{i}$ is the only prime divisor of $n$ with exponent 1 . Then $n=p_{1}^{\alpha_{1}} \cdot p_{2}^{\alpha_{2}} \ldots \ldots p_{i-1}^{\alpha_{i-1}} \cdot p_{i} \cdot p_{i+1}^{\alpha_{i+1}} \ldots \ldots . p_{k}^{\alpha_{k}}$.

Let $D=\left\{p_{1}, p_{2}, \ldots \ldots, p_{i-1}, p_{i} p_{j}, p_{i+1}, \ldots, p_{k}\right\}$. The vertex $p_{i}$ is adjacent only with the vertex $p_{i} p_{j}$ in $D$, for any $j$, as $G C D\left(p_{i}, p_{i} p_{j}\right)=p_{i}$. Now the degree of $p_{i}<$ the degree of $p_{i} p_{j}$.Hence it follows as in Case 1 that the vertices in $D$ strongly dominate the vertices of $V-D$.

Therefore $D=\left\{p_{1}, p_{2}, \ldots, p_{i-1}, p_{i} p_{j}, p_{i+1}, \ldots, p_{k}\right\}$ is a strong dominating set of $G\left(V_{n}\right)$ with cardinality $k$.

Hence $\gamma_{s}\left(G\left(V_{n}\right)\right)=k$.

Case 3: Suppose $\alpha_{i}=1$ for more than one $i$.

We have the following sub cases.

Sub case (i): Suppose $\alpha_{i}=1$ for all $i$. That is

$n=p_{1} p_{2} \ldots \ldots p_{k}$.

Here we have the following possibilities.

Suppose $k=2$. Then the vertex set of $G\left(V_{n}\right)$ is $\left\{p_{1}, p_{2}, p_{1} p_{2}\right\}$. Obviously $D=\left\{p_{1} p_{2}\right\}$ is a dominating set of $G\left(V_{n}\right)$ and this set is also a strong dominating set with cardinality 1 as $\operatorname{deg}\left(p_{1} p_{2}\right)>\operatorname{deg}\left(p_{1}\right), \operatorname{deg}\left(p_{2}\right)$.

Suppose $k=3$.

Then $V\left(G\left(V_{n}\right)\right)=\left\{p_{1}, p_{2}, p_{3}, p_{1} p_{2}, p_{1} p_{3}, p_{2} p_{3}, p_{1} p_{2} p_{3}\right\}$. It is clear that $D=\left\{p_{1}, p_{2} p_{3}\right\}$ is a strong dominating set of $G\left(V_{n}\right)$, as $\operatorname{deg}\left(p_{2} p_{3}\right)>\operatorname{deg}\left(p_{2}\right), \operatorname{deg}\left(p_{3}\right)$ and $\operatorname{deg}\left(p_{2} p_{3}\right)=$ $\operatorname{deg}\left(p_{1} p_{3}\right), \operatorname{deg}\left(p_{1} p_{2}\right) ; \operatorname{deg}\left(p_{1}\right)=\operatorname{deg}\left(p_{1} p_{2} p_{3}\right)$.

Therefore $D$ is a strong dominating set of $G\left(V_{n}\right)$ with cardinality 2 .

Suppose $\mathrm{k}>3$. Now we show that the set $D=\left\{p_{1}, p_{1} p_{2}, p_{2} p_{3}, \ldots, p_{k-3} p_{k-2}, p_{k-1} p_{k}\right\}$ is a dominating set of $G\left(V_{n}\right)$. In this case the vertices of $G\left(V_{n}\right)$ are primes $p_{1}, p_{2}, \ldots \ldots, p_{k}$ and their products.
All the vertices $u \in V-D$, for which $\operatorname{GCD}\left(u, p_{1}\right)=p_{1}$ are adjacent with the vertex $p_{1}$ in $D$, the vertices $p_{2}, \ldots, p_{k}$ are adjacent with the vertices $p_{1} p_{2}$ and $p_{2} p_{3} ; p_{2} p_{3}$ and $p_{3} p_{4} ; \ldots ; p_{k-4} p_{k-3}$ and $p_{k-3} p_{k-2} ; p_{k-1} p_{k}$ respectively.

The vertices

$p_{2} p_{3}, p_{2} p_{4}, p_{2} p_{5}, \ldots \ldots, p_{2} p_{k}, p_{2} p_{3} p_{4}, \ldots \ldots, p_{2} p_{3} p_{k}$ and so on are adjacent with the vertex $p_{1} p_{2}$, the vertices

$p_{3} p_{1}, p_{3} p_{4}, p_{3} p_{5}, p_{3} p_{6}, \ldots \ldots, p_{3} p_{k}, p_{3} p_{4} p_{5}, \ldots \ldots, p_{3} p_{4} p_{k}$ and so on are adjacent with the vertex $p_{2} p_{3}$.

Similarly we can show that every vertex in $V-D$ shares a common factor $p_{1}$ or $p_{2}$ or ...... or $p_{k}$ with some vertex in $D$

Thus every vertex in $V-D$ is adjacent with at least one vertex in $D$.

Therefore $D$ becomes a dominating set of $G\left(V_{n}\right)$.

By the definition of edges in $G\left(V_{n}\right)$, we see that $\operatorname{deg}\left(p_{i} p_{j}\right)>\operatorname{deg}\left(p_{i} p_{j} p_{l}\right)>\cdots>\operatorname{deg}\left(p_{1} p_{2} p_{3} \ldots p_{k}\right) ;$ $\operatorname{deg}\left(p_{i}\right)<\operatorname{deg}\left(p_{i} p_{j}\right) ; \operatorname{deg}\left(p_{i}\right)>\operatorname{deg}\left(p_{i} p_{j} p_{l}\right), \ldots, \operatorname{deg}\left(p_{i}\right)>$ $\operatorname{deg}\left(p_{1} p_{2} p_{3} \ldots p_{k}\right), i=1,2, \ldots, k$. Now the vertex $p_{1} p_{2} \ldots \ldots p_{k}$ is adjacent with the vertex $p_{1} \in D$ only.

Thus every vertex in $V-D$ is strongly dominated by at least one vertex in $D$.

Therefore $D=\left\{p_{1}, p_{1} p_{2}, p_{2} p_{3}, \ldots \ldots, p_{k-3} p_{k-2}, p_{k-1} p_{k}\right\}$ is a strong dominating set of $G\left(V_{n}\right)$.

Now we show that $D$ is minimum. Suppose we delete the vertex $p_{i}$ from $D$, then the vertex $p_{1} p_{2} \ldots p_{k}$ is not adjacent with any other vertex in $D$, because $G C D\left(p_{1} p_{2}, p_{1} p_{2} \ldots p_{k}\right)=$ $p_{1} p_{2} ; C D\left(p_{2} p_{3}, p_{1} p_{2} \ldots \ldots \ldots p_{k}\right)=p_{2} p_{3} ; \ldots \ldots \ldots$; $G C D\left(p_{k-3} p_{k-2}, p_{1} p_{2} \ldots p_{k}\right)=p_{k-3} p_{k-2}$; $G C D\left(p_{k-1} p_{k}, p_{1} p_{2} \ldots \ldots p_{k}\right)=p_{k-1} p_{k}$.

Similar is the case with the deletion of any vertex $p_{i} p_{j}$ from $D$, for $i=1,2, . . k-3, k-1$;

$j=2,3, \ldots . k-2, k$.

Thus $D=\left\{p_{1}, p_{1} p_{2}, p_{2} p_{3}, \ldots, p_{k-3} p_{k-2}, p_{k-1} p_{k}\right\}$ is a minimum strong dominating set of $G\left(V_{n}\right)$.

Hence $\gamma_{s}\left(G\left(V_{n}\right)\right)=k-1$.

Sub case (ii): Suppose $\alpha_{1}=\alpha_{2}=1$. That is $n=p_{1} \cdot p_{2} \cdot p_{3}^{\alpha_{3}} \cdot p_{k}^{\alpha_{k}}$.

Now we show that the set $D=$ $\left\{p_{3}, p_{4}, \ldots, p_{k}, p_{1} p_{2}\right\}$ becomes a dominating set of $G\left(V_{n}\right)$.

The vertices $p_{1}, p_{2}$ are adjacent only with the vertex $p_{1} p_{2}$ in $D$, as $G C D\left(p_{1}, p_{1} p_{2}\right)=p_{1} ; G C D\left(p_{2}, p_{1} p_{2}\right)=p_{2}$. Now $\operatorname{deg}\left(p_{1} p_{2}\right)>\operatorname{deg}\left(p_{1}\right), \operatorname{deg}\left(p_{2}\right)$, Hence it follows as in Case 1 that the vertices in $D$ strongly dominate the vertices of $V-D$.

Therefore $D=\left\{p_{3}, p_{4}, \ldots \ldots, p_{k}, p_{1} p_{2}\right\}$ is a strong dominating set of $G\left(V_{n}\right)$ with cardinality $k-1$.

Hence $\gamma_{s}\left(G\left(V_{n}\right)\right)=k-1$

Sub case (iii): Suppose $\alpha_{1}=\alpha_{2}=\alpha_{3}=1$. That is

$n=p_{1} \cdot p_{2} \cdot p_{3} p_{4}^{\alpha_{4}} \ldots \ldots p_{k}^{\alpha_{k}}$. 
Let $D=\left\{p_{4}, p_{5}, \ldots \ldots, p_{k}, p_{1}, p_{2} p_{3}\right\}$. Then we can show as in Subcase (ii) that $D$ is a strong dominating set of $G\left(V_{n}\right)$ with cardinality $k-1$.

Hence $\gamma_{s}\left(G\left(V_{n}\right)\right)=k-1$.

Sub case (iv): Suppose $\alpha_{\mathrm{j}}=1$ for $j=1,2, \ldots \ldots, i$ and $\alpha_{\mathrm{j}}>1$ for $j=i+1, i+2, \ldots . ., k$.

Then $n=p_{1} \cdot p_{2} \cdot p_{3} \ldots \ldots p_{i} \cdot p_{i+1}^{\alpha_{i+1}} \ldots \ldots p_{k}^{\alpha_{k}}$.

We can prove as in Case 1 and Sub case (i) of Case 3 that the set $D=\left\{p_{i+1}, p_{i+2}, \ldots, p_{k}, p_{1}, p_{1} p_{2}, \ldots, p_{i-3} p_{i-2}, p_{i-1} p_{i}\right\}$ forms a minimum strong dominating set of $\left(V_{n}\right)$.

Hence $\gamma_{s}\left(G\left(V_{n}\right)\right)=k-1$.

\section{CONCLUSION}

Using Number theory, it is interesting to study the strong dominating sets of these Arithmetic graphs. This work gives the scope for the study of strong dominating sets of product graphs of these graphs and the authors have also studied this aspect.

\section{ILLUSTRATIONS}

\section{Euler totient Cayley Graphs}

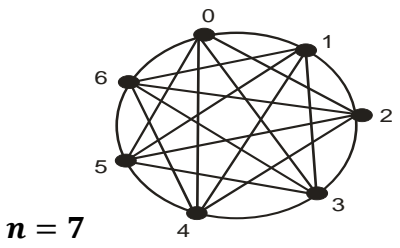

Fig.1

$G\left(Z_{7}, \varphi\right)$

Strong Dominating Set $=\{0\}$.

$n=14=2 \times 7$

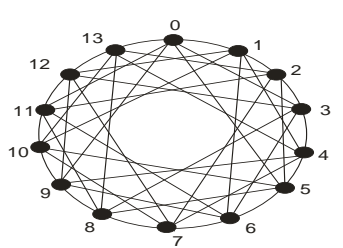

Fig.2

$G\left(Z_{14}, \varphi\right)$

Strong Dominating Set $=\{0,7\}$.

$n=8=2^{3}$

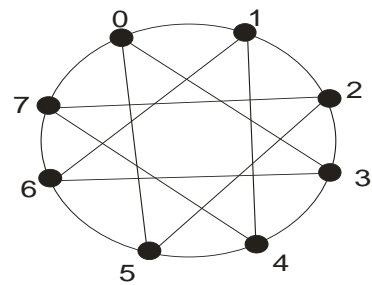

Fig.3

$G\left(Z_{8}, \varphi\right)$

Strong Dominating Set $=\{0,1\}$. $n=100=2^{2} \times 5^{2}$

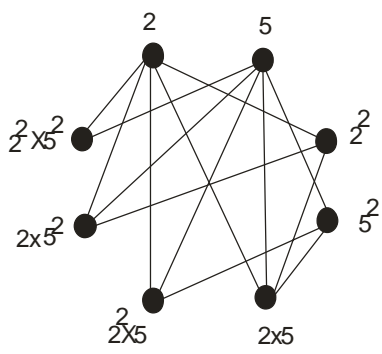

Fig.4

$\mathbf{G}\left(\mathbf{V}_{\mathbf{1 0 0}}\right)$

Strong Dominating Set $=\{2,5\}$.

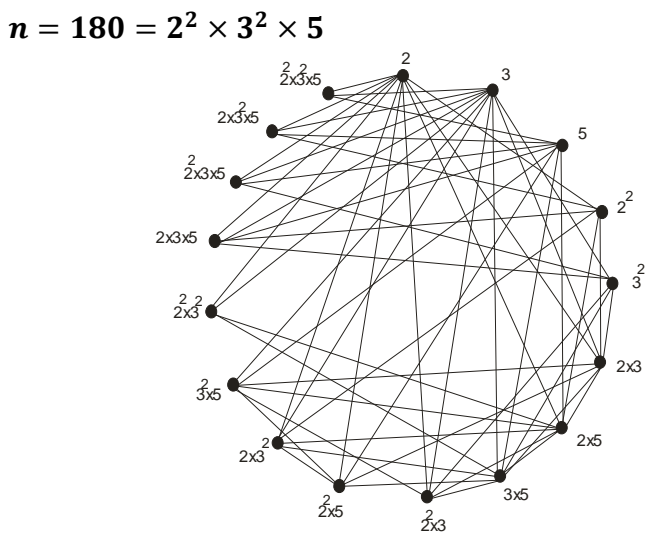

Fig.5

$\mathbf{G}\left(\mathbf{V}_{\mathbf{1 8 0}}\right)$

Strong Dominating Set $=\{2,3,15\}$

$n=10=2 \times 5$

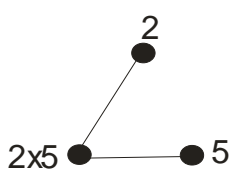

Fig.6

$G\left(V_{10}\right)$

Strong Dominating Set $=\{10\}$.

$n=30=2 \times 3 \times 5$

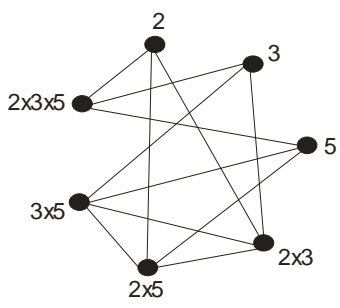

Fig.7

$G\left(V_{30}\right)$

Strong Dominating Set $=\{2,15\}$.

$n=210=2 \times 3 \times 5 \times 7$ 


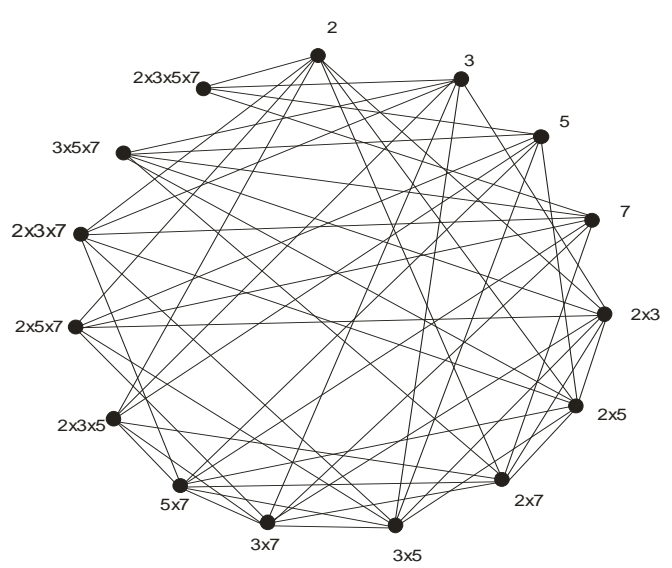

Fig.8 $G\left(V_{210}\right)$

Strong Dominating Set $=\{2,6,35\}$.

$n=60=2^{2} \times 3 \times 5$

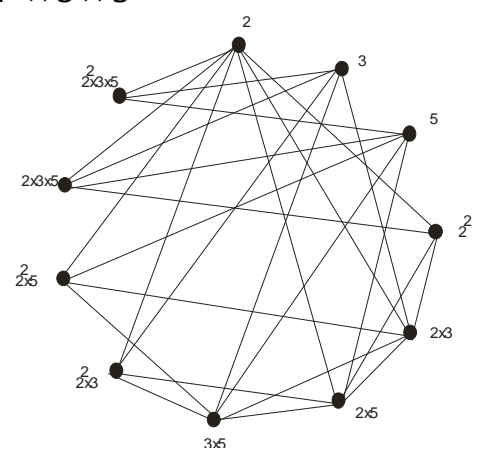

Fig.9

$\boldsymbol{G}\left(\boldsymbol{V}_{60}\right)$

Strong Dominating Set $=\{2,15\}$.

$n=420=2^{2} \times 3 \times 5 \times 7$

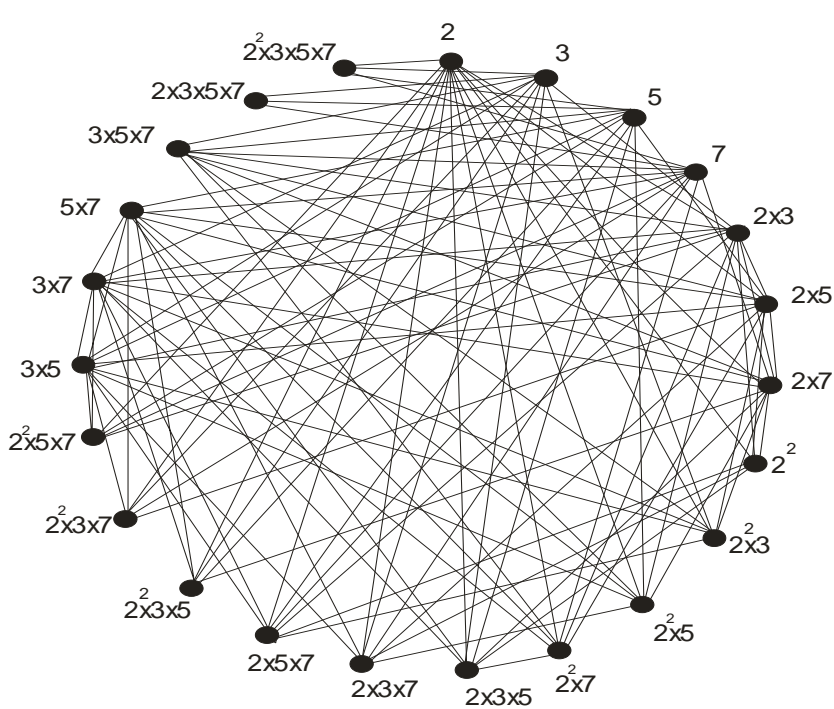

Fig.10

$$
\boldsymbol{G}\left(\boldsymbol{V}_{\mathbf{4 2 0}}\right)
$$

Strong Dominating Set $=\{2,3,35\}$.

\section{REFERENCES}

[1] Nathanson and B.Melvyn, -Connected components of arithmetic graphs, Monat.fur.Math, 29, (1980), 219 220.

[2] L.Madhavi, Studies on domination parameters and enum eration of cycles in some Arithmetic Graphs,Ph.D. Thesi s submitted to S.V.University, Tirupati, India, (2002).

[3] S.Uma Maheswari, and B.Maheswari, - Domination parameters of Euler Totient Cayley Graphs, Rev.Bull.Cal Math.Soc., 19 (2), (2011), 207-214.

[4] S.Uma Maheswari, and B.Maheswari, - Some Domination parameters of Arithmetic Graph Vn, IOSR,J ournal of Mathematics, Volume 2, Issue 6, (Sep Oct 201 2), $14-18$

[5] E.Sampathkumar, L. Pushpa Latha - Strong weak domination and domination balance in graph, Discrete Mathematics, 161(1996), 235-242. 